\title{
A Rare Case of Peritoneal Mice Presenting as Acute Abdomen
}

\author{
Dr.A.Jamila, Dr. S. Ravi, Dr. K. Valarmathi, Dr.Selvambigai
}

\begin{abstract}
Peritoneal loose bodies or peritoneal mice are asymptomatic incidentalomas predominantly found at abdominal surgery orat autopsy. Peritoneal loose bodies evolve from torsion and separation of appendices epiploicae. Peritoneal loose body is a histopathological diagnosis and most other differential diagnosis for calcified intra peritoneal masses can be ruled out during the histological examination.
\end{abstract}

Key Words: Calcified mass, Peritoneal loose body, Mice, Acute abdomen.

\section{Introduction}

Peritoneal loose body or mice are very rare and only a few cases have been reported. Many lesions are discovered incidentally during abdominal surgery or autopsy.

\section{Case Report}

A 53 years-old male patient presented with acute and severe abdominal pain, diarrhoea and vomiting of 5 days duration. Per abdominal examination revealed a palpable freely mobile mass in the right iliac fossa. CT examination showed a calcified mass in themesentery. Patient was operated by mid line incision and a pearly white egg like mass lying freely between intestine, in peritoneal cavity was found. When tried for removal of the mass, it came out as a free mass in total, without any evidence of attachment or adherence to any part of intestine or mesentery.

\section{Macroscopic appearance:}

Received a single globular, glistening,slightly grey and pearly white mass measuring $6 \times 6 \times 4 \mathrm{~cm}$, weighing $64 \mathrm{gms}$. The mass was gritty to cut. Cut section appeared grey white with whorled appearance.

\section{Microscopic appearance:}

Sections studied from the mass showed strips of hyalinised collagenous material with blotchy and sprinkled calcification. The entire mass was acellular. No evidence of any normal histological structures in the material studied. Provisional impression was Hyaline mass with Dystrophic calcification.

\section{Discussion}

Peritoneal loose bodies are incidentalomas they measure about 0.5 to $2.5 \mathrm{cms}$ in diameter. Giant loose bodies measuring more than $5 \mathrm{cms}$ can be associated with symptomatology due to mass effect ${ }^{1}$. Most common cause or peritoneal loose bodies are thought to arise by torsion and separation of appendices epiploicae ${ }^{2}$.

Epiploic appendages referred as appendices epiploicae are $1-2 \mathrm{cms}$ thick and 0.5 to $5 \mathrm{cms}$ long, supplied by one or two small colonic end arteries and small draining vessels ${ }^{3}$. The small physiological peritoneal fat pouches are attached to the external surface of the colon by vascular stalk. In 1863, Virchow proposed the theory of formation of loose peritoneal bodies. He said that as a result of obesity, the appendices epiplocae increases in fat content and their weight increases which in turn may lead to gradual and progressive obstruction of blood vessels orit may lead to torsion of pedicle which results in infarction and amputation ${ }^{4}$ Patterson in 1933 suspected that ischemia as a result of torsion or inflammation is dominant etiological factor that led to infarction or amputation ${ }^{5}$. Peritoneum reacts by deposition of fibrinoid material over it and it increases in size. ${ }^{6}$ Torsion and infarction are more common in obese people in whom epiploicae are bulky. The right iliac fossa remains the most common site. Pain, tenderness and palpable right iliac fossa mass are the common symptoms reported. ${ }^{7}$

Peritoneal loose bodies have been described as cause for intestinal obstruction or urinary retention depending on their size and intra-abdominal localization. ${ }^{89}$ Peritoneal loose bodies are also found from subacute attacks of pancreatitis ${ }^{10}$. They typically have fat attenuation and cannot be distinguished from other adipose structure. Peritoneal loose bodies by CT imaging reveals, a concentric round or oval shaped well defined mass with central calcification surrounded by a peripheral soft tissue usually located in pelvis. Often the mass will have a distinct fat plane separating it. On MRI, peritoneal loose bodies appear as well circumscribed, low intensity mass on both $T_{1}$ and $T_{2}$ weighted images. The mass does not exhibit any enhancement, the lack of enhancement is expressed as there is no blood supply. ${ }^{11}$ 
Peritoneal loose body should be differentiated from desmoid tumor, teratoma, calcification of lymph node, metastatic lesion of ovarian cancer, leiomyoma, GIST or retained sponge. Differential diagnosis of mobile pelvic mass with central calcification would include retained sponge or loose body. Wide window width CT clearly showed a concentric calcification, characteristic feature of peritoneal loose body ${ }^{12}$. GIST exhibits a contrast enhancing effect unlike peritoneal loose body which requires additional prone position CT examination to monitor their mobility. Differential diagnosis of calcified intra-peritoneal mass can be ruled out by histopathologic examination of the mass.

\section{References}

[1]. Ghanremani GG, White EM, Hoff FL, Gore RM, and Matter, Christ ML- Appendices epiploicae of the colon: Radiologic and pathologic features, 1992:12:59-77.

[2]. RossJA,McQueenA, Peritoneal loose bodies. Br J surg, 1947/48:35:313 -7.

[3]. Ross JA: Vascular loops in appendices epiploicae: their anatomy and surgical significance with a surgical pathology of appendices epipolicae. Br.J Surgery $1950\{148\}: 464-4$.

[4]. Borg SA, Whitehouse, Griffiths: A mobile calcified amputated appendix epiploica: AM J Rentogenol, 1976:127:349-506.

[5]. Patterson DC, Appendices epiploicae and their surgical significance with report of 3 cases, N Eng. J med, 1933: 209; $1255-95$.

[6]. Tareq M, AL-jabber: Torsion of abdominal appendages presenting with acute abdominal pain, Annals of Saudi medicine, Vol 20.2010.

[7]. Ghost P, Strong C, Naugher W, Haghighi P: Peritoneal mice implicated in intestinal obstruction: report of a case and review of literature. Journal clinical gastroenterology, 200640 (5);427-430.

[8]. BhendwarAH,DesaiVV,GajbhijyeRN,DeshrajBP: Acute retention of urine due to loose peritoneal body, British journal of urology 1996; 78; (6) 951-952.

[9]. Short practice of surgery, Bailey and Love2 $6^{\text {th }}$ edition, p.no:981.

[10]. G. Gayer MD \& Petrovith MD: CT diagnosis of large peritoneal loose bodies; A case report and review of literature. British Journal of Radiology, 84(2011)e83-e8f

[11]. Ohgitani D, Kani H, Matsuki M, Kanazawa S, Narabavayashi I: A case ofgiant peritoneal loose body: usefulness of wide window width CT. NiponIgaku Hoshasen Gakkai Zasshi 2004, 64(4):223-224

CT Showing Calcified mass (Arrow)

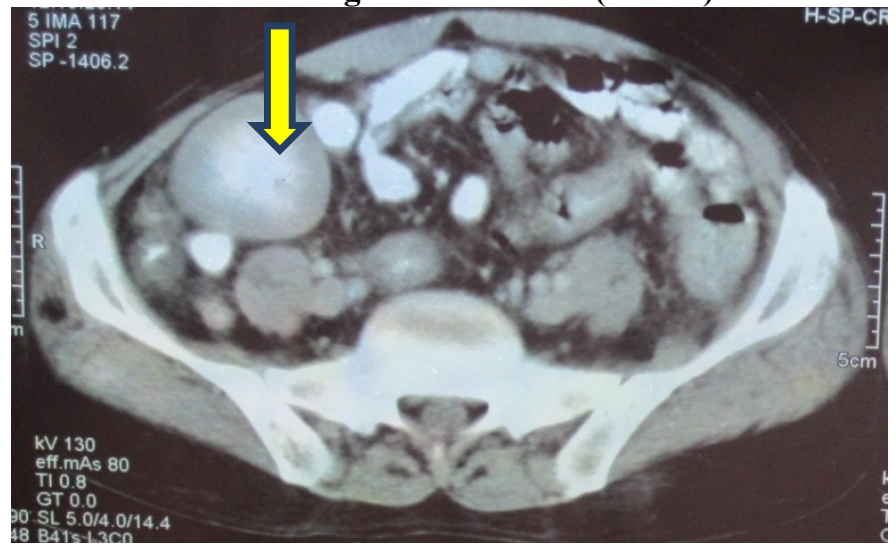

Per-operative finding showing a calcified mass

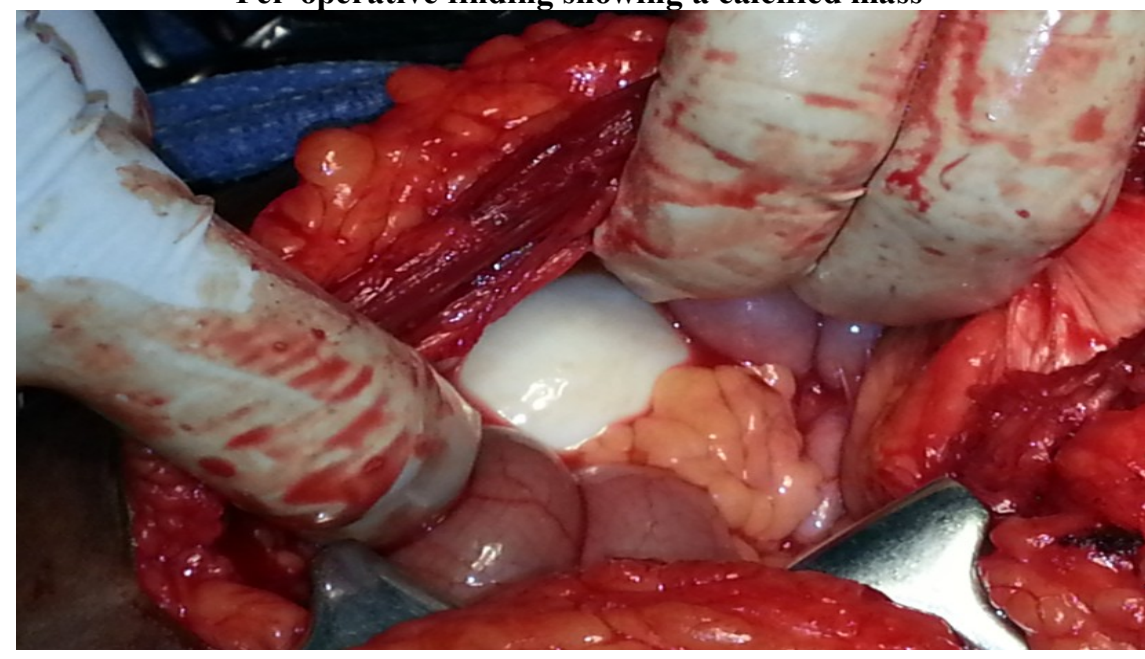


Intact mass removed during surgery

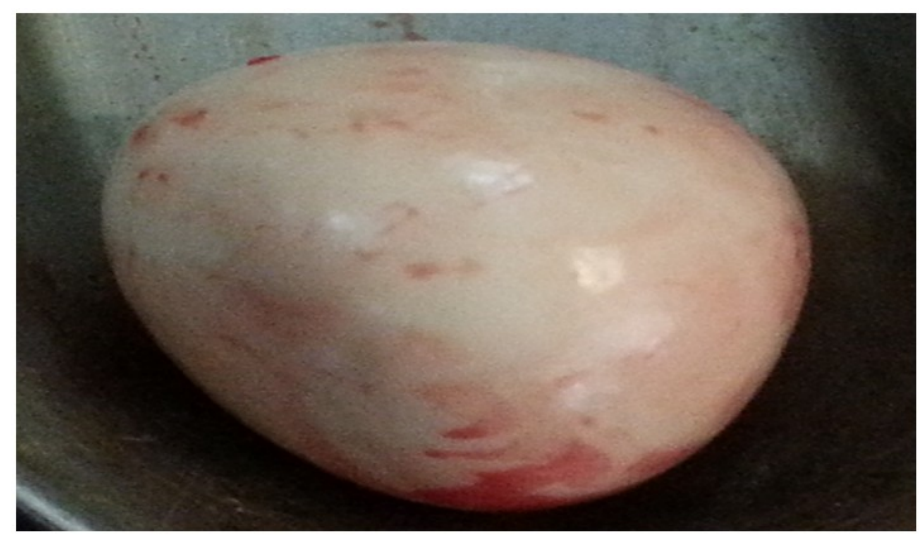

Smooth External Surface

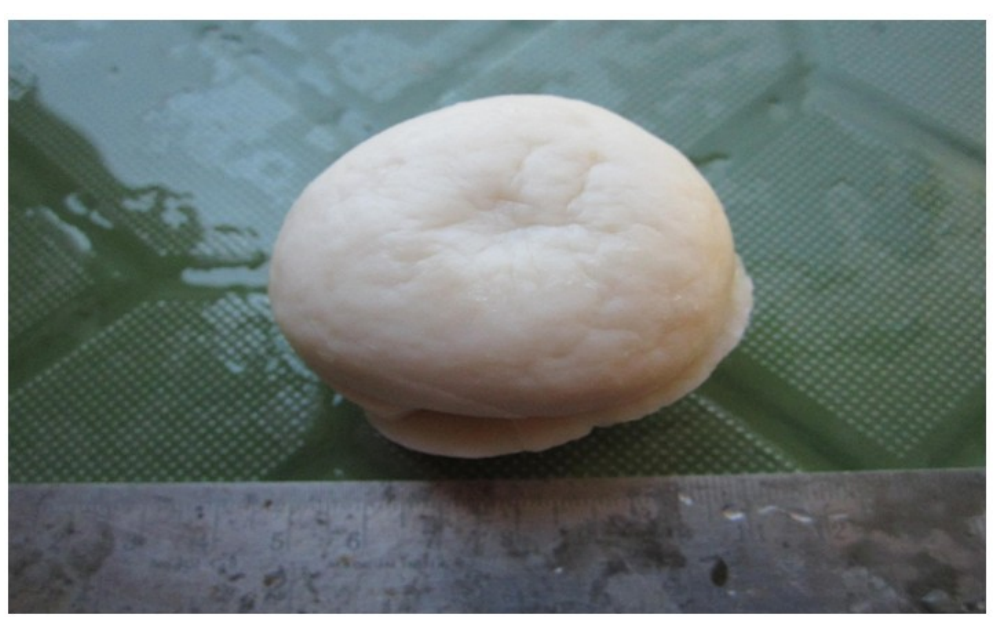

Cut Surface showing whorld Appearance

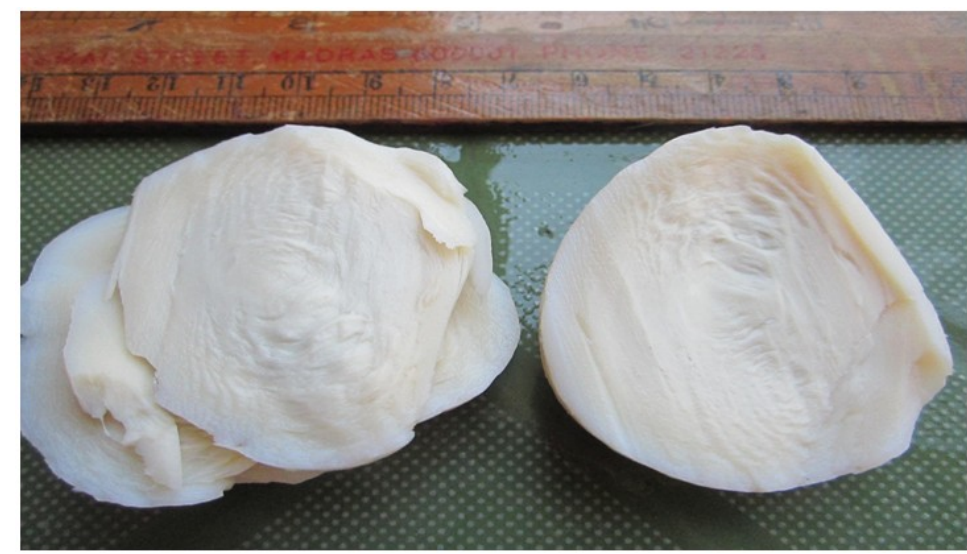


Microscopic picture showing Hyalinised fibrils

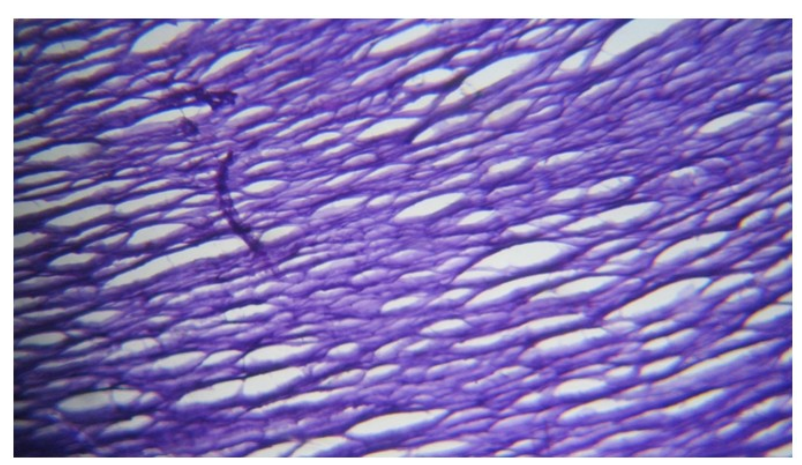

Microscopic picture showing foci of calcification

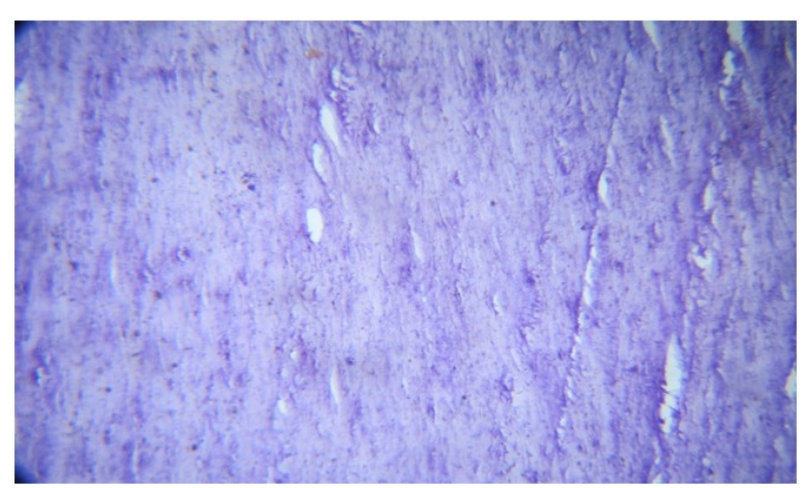

